\section{Another perspective on fasciculations: when is it not caused by the classic form of amyotrophic lateral sclerosis or progressive spinal atrophy?}

\author{
Marco Antonio Araujo Leite, ${ }^{1}$ \\ Marco Orsini,1 Marcos R.G. de Freitas, ${ }^{1}$ \\ João Santos Pereira,1,2 \\ Fábio Henrique Porto Gobbi, 3 \\ Victor Hugo Bastos, 4 \\ Dionis de Castro Machado, 4 \\ Sergio Machado, 5 Oscar Arrias-Carrion, 6 \\ Jano Alves de Souza, ${ }^{1}$ Acary Bulle \\ Oliveira ${ }^{7}$ \\ ${ }^{1}$ Neurology Service, Movement Disorders \\ Unit, Fluminense Federal University, \\ Niterói, Rio de Janeiro, Brazil; \\ ${ }^{2}$ Neurology Service, Rio de Janeiro \\ University, Brazil; ${ }^{3}$ Neurology \\ Department, São Paulo University, Brazil; \\ 4Departament of Physiotherapy, Piaui \\ Federal University, Parnaíba, Brazil; \\ 5Physical Activity Neuroscience, Physical \\ Activity Sciences Postgraduate Program, \\ Salgado de Oliveira University, Niterói, \\ Brazil; 6Movement Disorders and Sleep \\ Unit, General Hospital Dr. Manuel Gea \\ González, Secretaria de México D.F., \\ México; 7Neurology Service, São Paulo \\ Federal University, São Paulo, Brazil
}

\begin{abstract}
Fasciculations are visible, fine and fast, sometimes vermicular contractions of fine muscle fibers that occur spontaneously and intermittently. The aim of this article is to discuss the main causes for fasciculations and their pathophysiology in different sites of the central/peripheral injury and in particular to disprove that the presence of this finding in the neurological examination is indicative of amyotrophic lateral sclerosis. Undoubtedly, most fasciculations have a distal origin in the motor nerve both in normal subjects and in patients with motor neuron disease. Most of them spread to other dendritic spines often producing an antidromic impulse in the main axon. The clinical and neurophysiological diagnosis must be thorough. It may often take long to record fasciculations with electroneuromyography. In other cases, temporal monitoring is necessary before the diagnosis. The treatment, which may be adequate in some cases, is not always necessary.
\end{abstract}

\section{Introduction}

The fasciculations can be defined as visible fast, fine, spontaneous and intermittent contractions of muscle fibers. Some neurologists call them verminosis, because they look like worms moving below the dermis. ${ }^{1,2}$

Undoubtedly, this finding in a neurological examination is of concern, given its close relationship with amyotrophic lateral sclerosis (ALS), a disease that leads to a depletion of neurons in the fore tip and the pyramidal bundle. The presence of fasciculations, however, is not a sign of ALS when no other symptoms or signs of involvement of the fore tip and the pyramidal bundle are identified. ${ }^{3}$ No one knows whether the origin of ALS is central, in the first or second motor neurons, in the peripheral, or even in the terminal motor nerve - which is an even more intriguing aspect for experts. In short, it is incorrect to associate fasciculations directly to the injury of fore tip of the spinal cord, because the skeletal pathophysiological involvement can be quite broad. 3,4

Potential fasciculations may also persist after a distal nerve block. This suggests that fasciculation potentials may originate anywhere in the axon, probably within the distal axonal arborization. However they are known to be associated with a hypersensitivity of denervated muscles. It is also important to consider that several conditions may trigger them, such as other diseases of the fore tip of the spinal cord (benign monomelic amyotrophy, progressive spinal muscular atrophies, Hirayama disease and others), neuromuscular junction disorders, electrolyte disorders, systemic diseases and use of certain medications. ${ }^{5}$ Also healthy individuals can have fasciculations, although they are generally located in well-defined sites, such as the brachial distal third, the crural area and the eyelids. ${ }^{1}$ With regard to fasciculations potentials induced by drugs, Masland and Wigiton, ${ }^{6}$ in a pioneering experimental study, concluded that neostigmine can cause fasciculation potentials by increasing the concentration of acetylcholine at the neuromuscular junction in felines. Discharges are caused by the direct effect of acetylcholine in the motor nerve terminals. A similar mechanism also occurs during induction of anesthesia with succinylcholine endotracheally. ${ }^{7}$ Orsini et al. ${ }^{5}$ reported the case of a young man with benign fasciculations, triggered by the use of oral corticosteroids administered in immunosuppressive doses in patients with immune-mediated kidney disease, which subside completely after medication tapering.

The objective of this study based on the current literature is to describe various causes of fasciculations and to discuss the pathophysiological skeletal involvement, when present (Table 1).
Correspondence: Marco Antonio Araujo Leite, Fluminense Federal University - UFF Niterói, Rio de Janeiro, Brazil.

E-mail: maaraujoleite@yahoo.com.br

Key words: fasciculations, neurological diseases, electromyography treatment.

Contributions: the authors contributed equally.

Conflict of interests: the authors declare no potential conflict of interests.

Received for publication: 29 November 2013 Accepted for publication: 28 March 2014

This work is licensed under a Creative Commons Attribution NonCommercial 3.0 License (CC BYNC 3.0).

(C) Copyright M.A.A. Leite et al., 2014

Licensee PAGEPress, Italy

Neurology International 2014; 6:5208

doi:10.4081/ni.2014.5208

\section{Methods}

We reviewed several articles using the following databases: Lilacs, Scielo, Medline and Pubmed. In our search we used the following keywords: fasciculations, epidemiology, etiology, benign fasciculations syndrome, exercises, amyotrophic lateral sclerosis, motor neuron diseases, neuromuscular diseases, movement disorders, drugs, poisoning, physical activity and their correspondents in Portuguese, Spanish, Japanese in the period between 1940 and 2013.

\section{Fasciculations in healthy subjects}

Fasciculations can also be found in individuals with no neurological disease. In 1963 , Reed and Kurland warned that the presence of fasciculations was not necessarily a prelude to the onset of a progressive and lethal disease, due to the involvement of the lower motor neuron. ${ }^{8}$ Since then, several authors have explored this topic, defining a benign fasciculation syndrome (BFS), that most frequently affects young healthcare professionals, 9,10 who, in some cases, have already developed dyspnea. ${ }^{11}$ An interesting Australian prospective study published recently examined the cases of 20 physicians (20 consecutive cases) complaining of fasciculations. ${ }^{9}$ Fourteen of them were very concerned about being diagnosed with ALS. The fasciculations were mainly in the lower limbs, which had normal muscle strength. In the electrophysiological study, fas- 
ciculations potentials were of the simple type, motor conduction was normal and no signs of denervation or neurogenic changes of motor units were apparent.

These authors, in agreement with others, concluded that physical exercise, stress, fatigue and caffeine abuse can precipitate or aggravate this picture. Among the other six individuals in the sample, five patients manifested a cramp-fasciculation syndrome (Denny-Brown syndrome) and only one suffered from ALS.

Some authors have stated that, in order to establish the clinical diagnosis of BFS, a minimum of five years is necessary, due to the evolution, in some cases, of the motor neuron disease. $12-14$

A work by Fermont et al. ${ }^{15}$ reported the prevalence and distribution of fasciculations in healthy adults. The potentials were studied by ultrasound in 58 individuals from different age groups. The subjects were also interviewed using questionnaires about the exacerbation of caffeine consumption and physical activity. Of the total sample, $43 \%$ had fasciculations, especially in the hallucis longus abductor muscle. In the lower limbs, fasciculations were rarely found and reported. Older individuals showed more fasciculations than young adults. The authors have noted that certain physical activities, when very intense, may exacerbate symptoms in the lower limbs.

\section{Fasciculations associated with motor disorders}

Although fasciculations are not classified as a motor disorder, they are conceptually abnormal movements. Despite they are not rare, only a few studies investigate the association between fasciculations and motor disorders. ${ }^{16}$

In the cerebellum medullary degeneration type 3 (Machado-Joseph disease, SCA 3), a dominant autosomal disease related to an unstable and increased expansion of the CAG trinucleotide, is often associated with cramps, fasciculations and amyotrophy. ${ }^{17}$

In a series of 50 cases of SCA 3 France $e t$ $a l .18$ observed fasciculations in half of patients, most commonly in the facial region. Potential fasciculations were noticed in conventional electromyography in 41 cases. The authors reported the involvement of the peripheral nerves.

Kanai and Kuwabara posited that in SCA 3 the severity of cramps/fasciculations correlates with peripheral axonal excitability, 19 suggesting that the appearance of potentials is associated with subjacent axonal peripheral depletion and attempted sprouting of the remaining motor neurons for reinnervation of partially denervated muscles. Another cerebellar degeneration in which the ataxia is associated with fasciculations is SCA 36 . Recently described in Japanese families by Kobayashi et al., it is characterized by an increasing expansion of the hexanucleotide GGCCTG repeat in the first intron of the NOP56 gene. ${ }^{20}$

Ikeda et al. ${ }^{21}$ found that amyotrophy and fasciculations affected the tongue of most of the 18 SCA36 patients, and in more than half of them, trunk and limbs were also affected. They detected the involvement of the lower motor neuron using electroneuromyography (ENMG). In the same period, García-Murias et $a{ }^{22}$ described SCA 36 in two families from the region of Galicia in Spain. However, the observed phenotype differed from the Japanese one, since it occurred in association with a cerebellar syndrome, a condition of sensorineural hearing loss and restriction of movement like fasciculation or myokymia, in addition to (small) amyotrophy in the tongue. According to the previous description of Arias et al., SCA has been present in Spain since the 17th century, when such cases were discovered in the Galician village of Death Coast.

In certain situations, fasciculation potentials are associated with parkinsonisms. There are few reports of cases where such correlation is highlighted in the context of an atypical form of parkinsonism called multiple system atrophy (MSA). In an original article describing the Shy-Drager syndrome, ${ }^{23}$ a type of MSA, the authors reported the coexistence, in the two cases described, of fasciculations with dysautonomia, parkinsonism, cerebellar syndrome and other manifestations.
Montagna et al. ${ }^{24}$ reported fasciculations and amyotrophy in five cases of Shy-Drager syndrome. Clinical, electrophysiological, and anatomopathological examinations indicated the degenerative involvement of spinal previous ends.

In 2008, Luo et al. 25 described the case of a 68-year-old man, whose manifestations were muscle weakness, amyotrophy, fasciculations, tremor and cerebellar syndrome. The ENMG was consistent with spinal muscular atrophy (SMA) and olivopontocerebellar atrophy (OPCA), a type of MSA, which was confirmed by the MRI results. As there were no other cases in the family of the patient, the authors reported this to be the first case of SMA with sporadic OPCA. Unlike fasciculations, these are more frequent in patients with MAS, myoclonus, myokymia, and involved particularly the face. 26

Machida et al..$^{27}$ reported a case of sporadic amyotrophic lateral sclerosis with multiple system degeneration found at necropsy. A 48-yearold man who died one year after the onset of the disease had muscular atrophy, weakness and fasciculations in various body segments, ataxia and an increase of deep tendon reflexes in all four limbs in addition to rigidity and tremor. The necropsy showed a degeneration of the upper and lower motor neurons, the substantia nigra, the dentate nucleus and the locus coeruleus.

Under certain situations, fasciculations are associated with parkinsonisms. However, this link cannot be considered in the presence of at least two aspects. The distinction between hypertonia is not always an easy task in clinical practice. Spasticity and rigidity can occur at the same time in a patient.

The pyramidal damage can be so intense that it is not possible to verify a plastic hypertonicity, even when this results from a severe impairment of the substantia nigra. ${ }^{28}$ The other aspect is the bureaucratic interpretation of an excellent consensus for the diagnosis of amyotrophic lateral sclerosis, as the El Escorial revised, and diseases that are similar to parkinsonism, as can be seen in the Brain Bank of the Parkinson's Disease Society of the United Kingdom. 29,30

Table 1. Causes of fasciculations.

\begin{tabular}{ll}
$\begin{array}{l}\text { Fasciculationss in healthy subjects } \\
\text { Fasciculations associated with movement disorders }\end{array}$ & $\begin{array}{l}\text { Coffee; exhaustive physical activity/fatigue; stress; cramp syndrome and benign fasciculations } \\
\text { Espino-cerebell,ar degeneration-type 3; espino-cerebellar degeneration-type 36; parkinsonisms } \\
\text { (multiple system atrophy, ALS-plus syndromes) }\end{array}$ \\
\hline Motor neuron diseases & $\begin{array}{l}\text { Amyotrophic lateral sclerosis; progressive spinal muscular atrophies; benign monomelic amyotrophy; } \\
\text { Post-polio syndrome; Kennedy disease }\end{array}$ \\
$\begin{array}{ll}\text { Hystemic diseases } & \text { Hipertiteoidism; syndrome of inappropriate secretion of thyrotropin (Ohba); hypophosphatemia, } \\
\text { calcium disorders secondary to hyperparathyroidism }\end{array}$ \\
\hline Drugs and/or intoxications by heavy metals pollutants & $\begin{array}{l}\text { Neostigmine; corticosteroids; succinylcholine; elemental mercury intoxication; } \\
\text { association of lithium and nortriptyline; flunarizine; isoniazid }\end{array}$ \\
\hline
\end{tabular}

ALS, amyotrophic lateral sclerosis. 
Inaccurate reading of guidelines such as these can cause the elimination of different cases, due to their lack of consistency with these criteria. As a result there is a lack of studies and reports of non-classical cases. The first references concerning the coexistence of signs of involvement of both the upper and the lower motor neurons and the parkinsonian syndromes date back to almost a century ago. In 1923, van Bertrand and van Bogaert reported the pathological involvement of the basal ganglia in patients with ALS. In 1926, Alajouanine described the existence of motion disorders in rare cases of Charcot disease. Subsequently, in 1959, van Bondelle and colleagues presented a case of a woman who suffered from Parkinson's disease associated with ALS. ${ }^{31}$ More recent studies confirm the relationship between these disorders in experimental animals (transgenic mouse with superoxide dismutase - GH1) based on brain scintigraphy with an assessment of the dopaminergic transmission. ${ }^{28}$ Several syndromes, be them sporadic or hereditary, with the most diverse names have been described in relation to overlapping signs of parkinsonism, fasciculations and amyotrophy. Symptoms and signs of parkinsonism, usually bradykinesia and axial rigidity, occur in 5 to $17 \%$ of ALS patients and often respond badly to the use of levodopa. In these patients, brain scintigraphy shows a decreased striatal dopaminergic function, while neuropathological studies reveal a neuronal loss in the substantia nigra and in the globus pallidus. ${ }^{32}$ On the basis of El Escorial criteria they are classified as ALS-Plus Syndromes and ALS Mimic Syndromes in the presence of a parallel development of ALS and extrapyramidal signs. ${ }^{29}$ However, besides the fortuitous presence of extrapyramidal signs in the clinical course of some patients with ALS, it is likely that, in some conditions, PD and ALS develop in the same individual from the same pathogenic pathway.

Park and colleagues found a decrease in the transportation of dopamine in the putamen of two patients who showed parkinsonian signs and diffuse and progressive involvement in the upper and lower motor neurons. ${ }^{33}$ In both, bradykinesia, rigidity and tremor were asymmetrical and responsive to levodopa and dopamine agonist. Both manifested bilateral pyramidal signs and amyotrophy with diffuse fasciculations. In one, the signs of ALS preceded the PD ones, while in the other the opposite occurred. The authors also noticed that the decrease in dopamine transportation, determined by a pre-synaptic dysfunction of the nigrostriatal pathway was more pronounced in the contralateral striatum than the parkinsonian signs and reported such cases as ALS with parkinsonism (ALS-D). A recent multicentric study, ${ }^{31}$ which evaluated 6,471 with ALS, 7668 controls and 3146 with PD, found a correlation between gene variations in the gene for angiogenin in patients with ALS and signs of PD and increased risk of developing PD in relatives of patients with ALS and these variations. The authors suggest that these variations should be linked between the neurodegenerative process of ALS and PD. Brait, Fahn and Shwartz described three patients who showed an uncommon disease. ${ }^{34}$ It was observed in them the occurrence of symptoms and signs consistent with PD persistent to levodopa and the subsequent development (a few months to two years later) of ALS, in the absence of any other neurological signs or symptoms. Gilbert, Fahn, Mitsumoto and Rowland reviewed data from 5500 cases of parkinsonism documented by the Columbia University Division of Movement Disorders database and compared with data from Columbia University's MDA/ALS Research Center. ${ }^{3}$ As search words parkinsonism and upper motor neuron (UMN), lower motor neuron (LMN) dysfunction, or both were used. Twenty-five patients were identified. Two patients treated by Prof. Lewis P. Rowald were added. Out of these 27 cases in total, 7 had a form of ALS-p called by the authors Brait-Fahn disease, though with minor differences compared to the initial description, whereas in 3 cases there was a cognitive disorder and in 1 there were cerebellar changes. There were also 4 cases of Frontotemporal Dementia whith ALS, 6 cases of multiple system atrophy with ALS and 3 with a potential hereditary spastic paraplegia in which parkinsonism occurred with signs of involvement of LMN with or without a UMN disorder. The authors did not specify in how many of these patients fasciculations were assessed, given the fact that they are considered a sign of malfunction of the lower motor neuron, amyotrophy and/or fasciculation. Of the 23 cases with both parkinsonism and LMN signs, 11 showed an improvement with the use of levodopa. Manno et al. ${ }^{32}$ described 2 cases of Brait-Fahn-Schwartz disease in which progressive parkinsonian symptoms and signs preceded the appearance of ALS.

In these patients, SPECT with 123IIoflupane analysis revealed a bilateral reduced striatal uptake and genetic screening for S0D1, TDP-43, C9orf 72, FUS, angiogenin, Park-1, Park-2-Park and Park-6 7 (DJ-1) mutations were negative. In both the dopaminergic therapy (pramipexole and levodopa) was effective and long lasting in the treatment of Parkinsonian syndrome.

Annesi et al..$^{35}$ described a family from Southern Italy with a double homozygous mutation in the DJ-1 gene and a rather complex phenotype. In two of the patients described, amyotrophy and diffuse myofasciculations coexisted with bilateral piramidalism, bradykinesia, tremor at rest, rigidity. Some of them also had dementia. The order of appear- ance of motor neuron disease and parkinsonism varied in both patients. The authors named these cases as Early Onset Parkinson disease (EOPD)/ALS. Under the heading of neuromyotonias, there are reports of the coexistence of involuntary movements with fasciculations. This group is characterized by the occurrence of a failure of muscle relaxation and continuous muscle fiber activity. The most well-knonwn is the Isaacs Syndrome. This rare syndrome has several causes, such as neoplastic diseases, immune-mediated diseases, including para-neoplastic, hereditary and degenerative diseases. Furthermore, it is referred to as canulopatia due to loss of the voltage-dependent potassium channels function (VGKCs) damaged by antibodies. The failure of VGKCs results in hyperexcitability of peripheral nerves and consequent continuous muscle fiber activity. Clinically, cramps are observed with fasciculations, continuous involuntary muscle activity (clinical myokymia), stiffness, delayed muscle relaxation and hyperhidrosis.

In the electroneuromyography, spontaneous discharges of motor unit potentials are detected during the needle test, which appear as generalized myokymia or neuromiotonic discharges.

In Morvan Syndrome (Morvan's fibrillary chorea), another neuromyotonia even more unusual in which an hyperactivity in the central nervous systems (mental confusion, disturbances mnésticos, hallucinations, insomnia and myoclonus) coexist with peripheral symptoms (neuromyokymias), the combination of fasciculations and movement disorder is controversial. There are few reports. Deymeer et $a l .{ }^{36}$ describe fasciculations and tremor in a case of Morvan Syndrome associated with thymoma, whereas Irani et al. ${ }^{37}$ do not mention fasciculation in any of the patients in a series of 29 cases with Morvan (11 manifested thymoma).

\section{Fasciculations in motor neuron diseases}

Numerous motor neuron diseases occur with fasciculations, ${ }^{38}$ such as Progressive Spinal Muscular Atrophies, Amyotrophic Lateral Sclerosis, Benign Monomelic Amyotrophy, Post-Polio Syndrome, Kennedy's disease, among others. With respect to ALS, there are few studies that describe the early changes in the motor unit and the pathophysiological aspects both in the initial stage and along the natural course of disease. ${ }^{39}$ For this purpose De Carvalho and Swash studied the neurophysiological aspects of the anterior tibialis muscle (TA) in 73 ALS patients during the disease progression. 40 of these, 61 had a nor- 
mal muscle strength (Medical Research Council: 5) in TA and 12 a moderate paresis (Medical Research Council: 4). We also evaluated the potential presence of fasciculations and fibrillations/sharp-waves (fibs-sw), and quantified MU potentials (MUPs) and jitter. ALS patients with a normal strength in the TA muscle were investigated in serial studies. Fasciculation potentials were recorded in the anterior tibialis muscle $(\mathrm{MRC}=5)$ in 21 patients with ALS and normal motor unit potentials. Longitudinal studies confirmed that the patients presenting fasciculation potentials as the only abnormality progressed to MUP instability before large motor unit potentials associated with fibs-sw were detected. The complexity of FPs in patients with weak TA muscle was greater than in the latter group. The authors consider the potential of an early marker in fasciculations (ALS), which undoubtedly anticipates instability of motor unit potentials or the re-innervation process. This finding can be seen in the early stages of the disease, in which axonal excitability is increased. With disease progression neuronal dysfunction occurs with loss of motor unit potentials and a transitional process of compensatory re-innervation.

Deficits in neuromuscular transmission are considered one of the most important signs in the pathophysiology of ALS. Among them fasciculation potentials are almost always identified during the clinical and neurophysiological examination, since, according to some authors, they originate in peripheral axons. It is postulated that the dysfunction of potassium channels in axons of ALS patients evokes hyperexcitability of the membrane (axonal), which show potential fasciculations. An important fact is that the magnetic cortical stimulation can evoke the same potential fasciculations, corroborating the idea that such hyperexcitability may be present in both with the depletion of spinal motor neurons as cortical (pyramidal tract). After analyzing 2681 fasciculation potentials in 17 patients with ALS, Hirota et al. ${ }^{41}$ also supports the theory that the origin of fasciculations is combined. According to the authors, supraspinal segments have contributed to the onset of the process. All these findings suggest that involvement affects both the central and the peripheral system in ALS ${ }^{42}$. Such considerations may explain in part why patients with peripheral nerve injuries andor first motor neuron lesions may present potential fasciculations, although not very frequently.

\section{Fasciculations induced by systemic diseases, drugs and/ or intoxication by heavy metals}

Thyroid disorders such as hyperthyroidism and inappropriate secretion of thyrotropin syndrome (Ohba) can also trigger fasciculations. Similarly, hypophosphatemia and calcium disorders secondary to hyperparathyroidism can sometimes cause the same. ${ }^{43}$ With regard to pharmacological treatments, in a pioneering experimental study Wigiton and Masland concluded that neostigmine may cause fasciculations potentials by increasing the concentration of acetylcholine in the neuromuscular junction in felines. ${ }^{6}$ Discharges are caused by the direct effect of acetylcholine on motor nerve terminals. Moreover, due to a similar mechanism, the same occurs during the induction of anesthesia with succinylcholine by endotracheal tube. ${ }^{7}$

Finelli described an interesting case under the title of Drug-Induced Creutzfeldt-Jakoblike Syndrome. ${ }^{44}$ An elderly manifested rapidly progressive dementia, postural tremor, gait instability, myoclonus and fasciculations caused by a combination of lithium and nortriptyline. Postural tremor, multifocal myoclonus, amyotrophy and fasciculations were also reported in a woman treated with topiramate for migraine without aura. Orsini et al. ${ }^{5}$ also presented a case of fasciculations caused by oral corticosteroids at immunosuppressive doses in patients with immune-mediated kidney disease.

Adams et al. described the case of a 54-yearold man who presented a ALS-like condition after a brief, but intense exposure to elemental mercury. After the fall of mercury serum levels, the clinic signs ceased completely. The toxicity of mercury should be considered not only in subjects with recent dysfunction of cells of the anterior horn of the spinal cord, but also with conditions like peripheral neuropathies of unknown origin, tremor, ataxia, and a range of psychiatric symptoms including confusion and depression. 46

Shinkeigaku et al. ${ }^{47}$ presented a case (21-year-old man) of anti VGKC-complex antibody associated with a disorder presenting severe pain and fasciculations that predominantly in a single upper extremity. Recently also isoniazid and flunarizine have been considered a potential source of fasciculations. ${ }^{48,49}$

\section{Treatment}

It is noteworthy that there is no specific treatment for fasciculations, as it is symptomatic. The use of antiepileptic drugs such as carbamazepine and phenytoin has shown a partial therapeutic response. Gabapentin can also be used generally at low doses from 300 to $600 \mathrm{mg} .{ }^{1}$ However, due to their benign and transitional nature, it is not always necessary to treat fasciculations. A recent study proposed a new approach to treat the Cramps Syndrome and Benign Fasciculations through the identification and correction of sleep apnea, which in most cases can be one of the triggers. ${ }^{50}$ Meanwhile, one should be aware of the diagnosis, rule out the possibility of other neurological diseases and look for less obvious causes or the presence of systemic diseases, sometimes decompensated, for example, thyroid disorders and electrolyte abnormalities. Also one should watch for adverse effects to drugs, whose correction and control will allow an adequate therapeutic response. Since no specific drug has yet been identified to treat these disorders, a regular clinical monitoring should be performed for some time before formulating the diagnosis. Considering the wide pathophysiological skeletal involvement in the genesis of fasciculations, first it is necessary to identify their origin and then the therapeutic target.

\section{References}

1. Buainain RP, Souza Moura L, Oliveira ASB. Fasciculations. Rev Neurocienc 2000;8:314.

2. Masland RL, Wigiton RS. Nerve activity accompanying fasciculations produced by prostigmin. J Neurophysiol 1940;3:269-75.

3. Mills KR. Characteristics of fasciculationss in amyotrophic lateral sclerosis and the benign fasciculations syndrome. Brain 2010;133:3458-69.

4. Kleine BU, Stegeman DF, Schelhaas HJ, Zwarts MJ. Firing pattern of fasciculationss in ALS: evidence for axonal and neuronal origin. Neurology 2008;70:353-9.

5. Orsini M, Sztajnbok FR, Oliveira AB, et al. Benign fasciculationss and corticosteroid use: possible association? An update. Neurol Int 2011;3:e11.

6. Masland RL, Wigiton RS. Nerve activity accompanying fasciculations produced by prostigmin. J Neurophysiol 1940;3:269-75.

7. Muravchick S, Burkett L, Gold MI. Succinycholine-induced fasciculationss and intragastric pressure during induction of anesthesia. Anesthesiology 1981;55: 180-3.

8. Reed DM, Kurland LT. Muscle fasciculations in a healthy population. Arch Neurol 1963;9:363-7.

9. Simon NG, Kiernan MC. Fasciculations anxiety syndrome in clinicians. $\mathrm{J}$ Neurol 2013;260:1743-7.

10. Forcelini CM, Rotta FT, Posenato N. Benign fasciculationss responsive to gabapentin. Arq Neuropsiquiatr 2007;65: 1015-7.

11. Rigby J, Holton A, Partridge J, Satchithananda D. Dyspnoea fasciculations syndrome: the clue is in the title. 
BMJ Case Rep 2012:2012.

12. Singh V, Gibson J, McLean B, et al. Fasciculationss and cramps: how benign? Report of four cases progressing to ALS. J Neurol 2011;258:573-8.

13. Blexrud MD, Windebank AJ, Daube JR. Long term follow-up of 121 patients with benign fasciculationss. Ann Neurol 1993;34:622-5.

14. Okuda B, Kodama N, Tachibana H, Sugita M Motor neuron disease following generalized fasciculationss and cramps. J Neurol Sci 1997;150:129-31.

15. Fermont J, Arts IM, Overeem S, et al. Prevalence and distribution of fasciculationss in healthy adults: effect of age, caffeine consumption and exercise. Amyotroph Lateral Scler 2010;11:181-6.

16. Arias M, Arias-Rivas S, Blanco-Arias P, et al. [SCA from the Costa da Morte: A new SCA. Description of the phenotype]. Neurologia 2008;23:628-9. [Article in Spanish]

17. Tan EK, Tong J, Pavanni R, et al. Genetic analysis of SCA 2 and 3 repeat expansions in essential tremor and atypical Parkinsonism. Mov Disord 2007;22:1971-4.

18. França MC Jr, D’Abreu A, Nucci A, LopesCendes I. Muscle excitability abnormalities in Machado-Joseph disease. Arch Neurol 2008;65:525-9.

19. Kanai K, Kuwabara S. Motor nerve hyperexcitability and muscle cramps in Machado-Joseph disease. Arch Neurol 2009;66:138-141.

20. Kobayashi H, Abe K, Matsuura T, et al. Expansion of Intronic GGCCTG hexanucleotide repeat in NOP56 causes SCA36, a type of spinocerebellar ataxia accompanied by motor neuron involvement. Am J Hum Genet 2011;89:121-30.

21. Ikeda Y, Ohta Y, Kobayashi H, et al. Clinical features of SCA36: a novel spinocerebellar ataxia with motor neuron involvement (Asidan). Neurology 2012;79:333-41.

22. García-Murias M, Beatriz Quintáns B, Arias M, et al. Costa da Morte ataxia is spinocerebellar ataxia 36: clinical and genetic characterization. Brain 2012:135; 1423-35.

23. Shy GM, Drager GA. A neurological syndrome associated with orthostatic hypotension: a clinical-pathologic study. Arch Neurol 1960;2:511-27.

24. Montagna P, Martinelli P, Rizzuto N, et al.
Amyotrophy in Shy-Drager syndrome. Acta Neurol Belg 1983;83:142-57.

25. Luo W, Ouyang Z, Guo Y, et al. Spinal muscular atrophy combined with sporadic olivopontocerebellar atrophy. Clin Neurol Neurosurg 2008;110:855-8.

26. Blunt SB, Khalil NM, Perkin GD. Facial myokymia in multiple system atrophy. Mov Disord 1997;12:235-8.

27. Machida Y, Tsuchiya K, Anno M, et al. Sporadic amyotrophic lateral sclerosis with multiple system degeneration: a report of an autopsy case without respirator administration. Acta Neuropathol 1999;98:512-5.

28. Le Forestier, N, Lacomblez L, Meininger V. Syndromes parkinsoniens et scléroselatéraleamyotrophique: Tauopathies, TDP-43 et mutations SOD. Rev Neurol (Paris) 2009;165:15-30.

29. Brooks BR, Miller RG, Swash M, et al. El Escorial revisited: revised criteria for the diagnosis of amyotrophic lateral sclerosis. Amyotroph Lateral Scler Other Motor Neuron Disord 2000;1:293-9.

30. Hughes AJ, Daniel SE, Kilford L, Lees AJ. Accuracy of clinical diagnosis of idiopathic Parkinson's disease: a clinicopathological study of 100 cases. J NeurolNeurosurg Psychiatry 1992;55:181-4.

31. van Es MA, Schelhaas HJ, van Vught PW, et al. Angiogenin variants in Parkinson disease and amyotrophic lateral sclerosis. Ann Neurol 2011;70:964-73.

32. Manno C, Lipari A, Bono V, et al. Sporadic Parkinson disease and Amyotrophic Lateral Sclerosis complex (Brait-FahnSchwartz Disease). J Neurol Sci 2013;326:104-6.

33. Park HK, Lim YM, Kim JS, et al. Nigrostriatal dysfunction in patients with amyotrophic lateral sclerosis and parkinsonism J Neurol Sci 2011;301:12-3.

34. Brait K, Fahn S, Schwarz GA. Sporadic and familial parkinsonismand motor neuron disease. Neurology 1973;23:990-002.

35. Annesi G, Savettieri G, Pugliese P, et al. DJ-1 mutations and parkinsonism-dementia-amyotrophic lateral sclerosis complex. Ann Neurol 2005;58:803-7.

36. Deymeer F, Akca S, Kocaman G, et al. Fasciculationss, autonomicsymptoms and limbic encephalitis: a thymoma-associated Morvan's-like syndrome. Eur Neurol 2005;54:235-7.
37. Irani SR, Pettingill P, Kleopa KA, et al. Morvansyndrome: clinical and serological observations in 29 cases. Ann Neurol 2012,72:241-55.

38. Krivickas LS. Amyotrophic lateral sclerosis and other motor neuron diseases. Phys Med Rehabil Clin N Am 2003;14:327-45.

39. Higashihara M, Sonoo M. [Electrodiagnosis of ALS]. Brain Nerve 2007;59:103141. [Article in Japanese].

40. de Carvalho M, Swash M. Fasciculations potentials and earliest changes in motor unit physiology in ALS. J Neurol Neurosurg Psychiatry 2013;84:963-8.

41. Hirota N, Eisen A, Weber M. Complex fasciculationss and their origin in amyotrophic lateral sclerosis and Kennedy's disease. Muscle Nerve 2000;23:1872-5.

42. Kohara N. Abnormal hyperexcitability in ALS. Rinsho Shinkeigaku 1999;39:61-4. [Article in Japanese].

43. Godoy JM, Oliveira MAR, Moraes Neto JBM, et al. Esclerose lateral amiotrófica esporádica. Critérios diagnósticos. Arq Neuropsiquiatr 1993;51:236-42.

44. Finelli PF. Drug-induced Creutzfeldt-Jakob like syndrome. J Psychiatr Neurosci 1992;17:103-5.

45. Alonso-Navarro H, Jiménez-Jiménez FJ. Reversible tremor, myoclonus, and fasciculationss associated with topiramate use for migraine. Clin Neuropharmacol 2006;29:157-9.

46. Adams CR, Ziegler DK, Lin JT. Mercury intoxication simulating amyotrophic lateral sclerosis. JAMA 1983;250:642-3.

47. Hara K, Watanabe 0, Shibano K, Ishiguro H. [A case of anti VGKC-complex antibody associated disorder presenting with severe pain and fasciculations predominant in unilateral upper extremity]. Rinsho Shinkeigaku 2012; 52(9):677-80. [Article in Japanese].

48. Kizilay F, Ekmekci B, Gungor H, et al. Flunarizine-induced fasciculations-myokymia. J Clin Neuromuscul Dis 2011; 12:246-7.

49. Chaouch N, Mejid M, Zarrouk M, et al. [Isoniazid-induced myopathy]. Rev Pneumol Clin 2011;67:354-8. [Article in French].

50. Reddy PL, Grewal RP. Resolution of muscle cramps and fasciculations with treatment of sleep apnea. J Clin Neuromuscul Dis 2009;11:66-7. 